\title{
CTXT: hacia un modelo de negocio posible para el periodismo digital independiente
}

\author{
CTXT: Towards a possible business model for \\ independent digital journalism
}

\author{
Israel Márquez; Cristina Peñamarín
}

Cómo citar este artículo:

Márquez, Israel; Peñamarín, Cristina (2020). “CTXT: hacia un modelo de negocio posible para el periodismo digital independiente". El profesional de la información, v. 29, n. 1, e290113.

https://doi.org/10.3145/epi.2020.ene.13

Artículo recibido el 22-02-2019

Aceptación definitiva: 08-10-2019

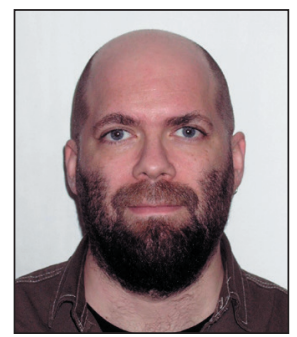

Israel Márquez $\bowtie$

https://orcid.org/0000-0001-7980-5570

Universidad Complutense de Madrid Facultad de Ciencias de la Información Avda. Complutense, 3

Ciudad Universitaria

28040 Madrid, España

isravmarquez@ucm.es

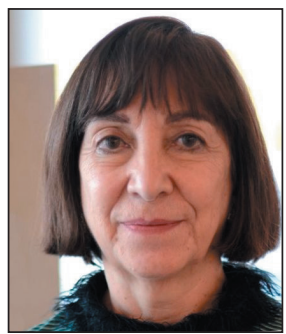

\author{
Cristina Peñamarín \\ https://orcid.org/0000-0002-5015-2235 \\ Universidad Complutense de Madrid \\ Facultad de Ciencias de la Información \\ Avda. Complutense, 3 \\ Ciudad Universitaria \\ 28040 Madrid, España \\ cpberis@ucm.es
}

\section{Resumen}

El presente trabajo tiene por objetivo profundizar en el conocimiento de CTXT, un semanario digital independiente de información general fundado en enero de 2015 por periodistas procedentes de diarios españoles de referencia y que en apenas cuatro años ha conseguido hacerse un hueco en el ecosistema mediático actual. A partir de una investigación etnográfica en curso sobre este nuevo cibermedio, nos centramos aquí en el análisis de su modelo de negocio, un aspecto central en el nacimiento y desarrollo de una pequeña empresa como CTXT, que emergió como una cuestión clave durante nuestro trabajo de campo. En un contexto de gran dificultad para levantar una nueva empresa periodística independiente, este modelo ha sido posible gracias a un continuo esfuerzo de búsqueda, experimentación y diversificación de las fuentes de ingresos y gracias a la pasión por el periodismo y al trabajo poco remunerado o gratuito de periodistas y otros profesionales muy cualificados. El esfuerzo del equipo se ha orientado a hacer productivo su importante capital simbólico (para construir la marca de un periodismo cualificado, independiente y alternativo al mainstream) y su capital social (sus redes con personas y con otros medios afines) para, pese a su reducido capital financiero, hacerse relevante en la esfera mediática. El periodismo que propone CTXT, atento a ser reflexivo, crítico y de calidad, ha sabido encontrar su nicho de audiencia y hacer oír su voz en la escena mediática.

\section{Palabras clave}

Periodismo; Periodismo digital; Prensa digital; Medios digitales; Cibermedios; Modelo de negocio; Financiación; Etnografía; Estudio de caso; CTXT.

\footnotetext{
Abstract

The aim of this paper is to deepen knowledge of CTXT, an independent digital weekly magazine founded in January 2015 by journalists from leading Spanish newspapers and that in just four years has managed to carve a niche in the current media ecosystem. Based on an ongoing ethnographic research, in this article we will focus on the analysis of its business model, a central aspect in the birth and development of a small company such as CTXT, which emerged as a key issue during our field work. In a context of great difficulty to start a new independent journalism company, this model has been possible thanks to a continuous effort of search, experimentation and diversification of sources of income and thanks to the passion for journalism and the low-paid or free work of journalists and other highly qualified professionals. The team's effort has been focused on making productive its important symbolic capital (to build the brand of a qualified, independent and alternative journalism) and its social capital (its networks with people and other related media)
} 
for, despite its small financial capital, become relevant in the media sphere. The type of journalism that CTXT proposes, attentive to being thoughtful, critical and of quality, has managed to find its audience niche and make its voice heard in the media scene.

\section{Keywords}

Journalism; Digital journalism; Online journlism; Digital press; Digital media; Cybermedia; Business model; Financing; Ethnography; Case study; CTXT.

\section{Introducción y estado de la cuestión}

CTXT es un semanario digital independiente fundado en enero de 2015 por periodistas procedentes de diarios españoles de referencia como El país y El mundo. En apenas cuatro años, el medio ha logrado ser sostenible económicamente y posicionarse en el ecosistema mediático español ocupando un espacio apenas presente en el mercado de medios digitales, el del semanario. A partir de este formato semanal y una apuesta decidida por recuperar un periodismo de calidad y "de servicio público", como lo denominan, CTXT ha conseguido atraer y afianzar a un amplio número de lectores durante su corta existencia.

Esta publicación forma parte de una nueva generación de cibermedios (eldiario.es, InfoLibre, etc.) nacidos en un contexto de crisis y metamorfosis del periodismo que ha modificado sustancialmente el escenario mediático (López-García, 2011) y está transformando la profesión en un conjunto dinámico de prácticas y expectativas

(Deuze; Witschge, 2017). Tras los ERES y reducciones de plantilla llevados a cabo a partir del año 2008 por muchos diarios, entre ellos, El país, El mundo o $A B C$, y la desaparición en febrero de 2012 de la edición en papel del diario Público (Fernández-Sande, 2013), varios periodistas despedidos de estos grandes medios de información general y sin ofertas de trabajo en un panorama de drástica reducción de plantillas, se vieron obligados a "reciclarse", implicándose en nuevos proyectos periodísticos o incluso creando nuevos diarios digitales. Esta situación dio lugar a una eclosión de medios digitales sin precedentes que guarda ciertas similitudes con la irrupción de nuevos medios durante la Transición, ya que, al igual que entonces, estos proyectos logran encontrar o crear un nicho de demanda:

"se apoyan en una demanda de información por parte de un significativo número de usuarios, cuya pretensión es resarcirse de las presiones de una preagenda mediática con sujeción a numerosos poderes fácticos" (García-Santamaría; Clemente-Fernández; López-Aboal, 2013, p. 143).

La crisis y los despidos se convirtieron así en un hervidero de nuevos medios digitales -muchos nacidos de la iniciativa y el trabajo, a menudo no remunerado, de los propios periodistas-, medios que están transformando el trabajo periodístico y las formas de pensarlo, imaginarlo, practicarlo y financiarlo (Peñamarín, 2016).

Esta vitalidad del periodismo digital forma parte de una tendencia global en nuestro entorno postindustrial: surgen nuevas empresas periodísticas, start-ups en red, creadas por profesionales cualificados y con experiencia que se encuentran en paro. Estas empresas generalmente pequeñas desarrollan modelos de negocio, formas organizativas y tácticas nuevas adaptadas a los usos y posibilidades de los entornos y recursos digitales. Y promueven una visión propia del periodismo. Según Deuze (2017), muchas de esas nuevas empresas se presentan como críticas con el statu quo del periodismo y afirman buscar en la Red la posibilidad de crear un medio que recupere el "verdadero" periodismo. Defienden sobre todo el principio de autonomía del periodismo respecto a los poderes económicos y políticos y su papel clave en democracia, su obligación de servir al público información independiente y de calidad. Esa pasión por su trabajo, que encuentran los investigadores en el estudio de veintiuna de estas start-ups de diferentes países (Deuze, 2017), permite a los profesionales afrontar condiciones laborales muy duras y exigentes, con bajas retribuciones y poca seguridad. A pesar de ello, manifiestan sentirse fuertemente vinculados a esas nuevas empresas donde trabajan de acuerdo a sus valores y donde pueden pertenecer a un equipo que comparte esa orientación.

En España, si bien medios como eldiario.es o InfoLibre han sido objeto de publicaciones científicas (González-Esteban, 2014; Rubio-Jordán, 2014; García-Orosa, 2016; López-García, 2016), en el caso de CTXT nos encontramos con una absoluta falta de estudios académicos sobre este novedoso medio digital, actor relevante en el actual panorama informativo. Este artículo es un intento de llenar este vacío académico.

\section{Objetivos y metodología}

La presente investigación tiene por objetivo profundizar en el conocimiento de un nuevo medio digital, CTXT, que defiende un periodismo crítico y de calidad y que aspira a la autosuficiencia económica, esto es, a la independencia como condición de libertad para el ejercicio de ese tipo de periodismo. Como veremos, CTXT ha contado con el compromiso de numerosos profesionales cualificados, con el capital inicial de sus socios fundadores, así como con el apoyo económico y el interés de sus lectores y suscriptores, pero la batalla por los recursos económicos sigue centrando buena parte de los esfuerzos de su equipo, de ahí que el problema del modelo de negocio emergiera como una cuestión clave durante nuestra investigación. 

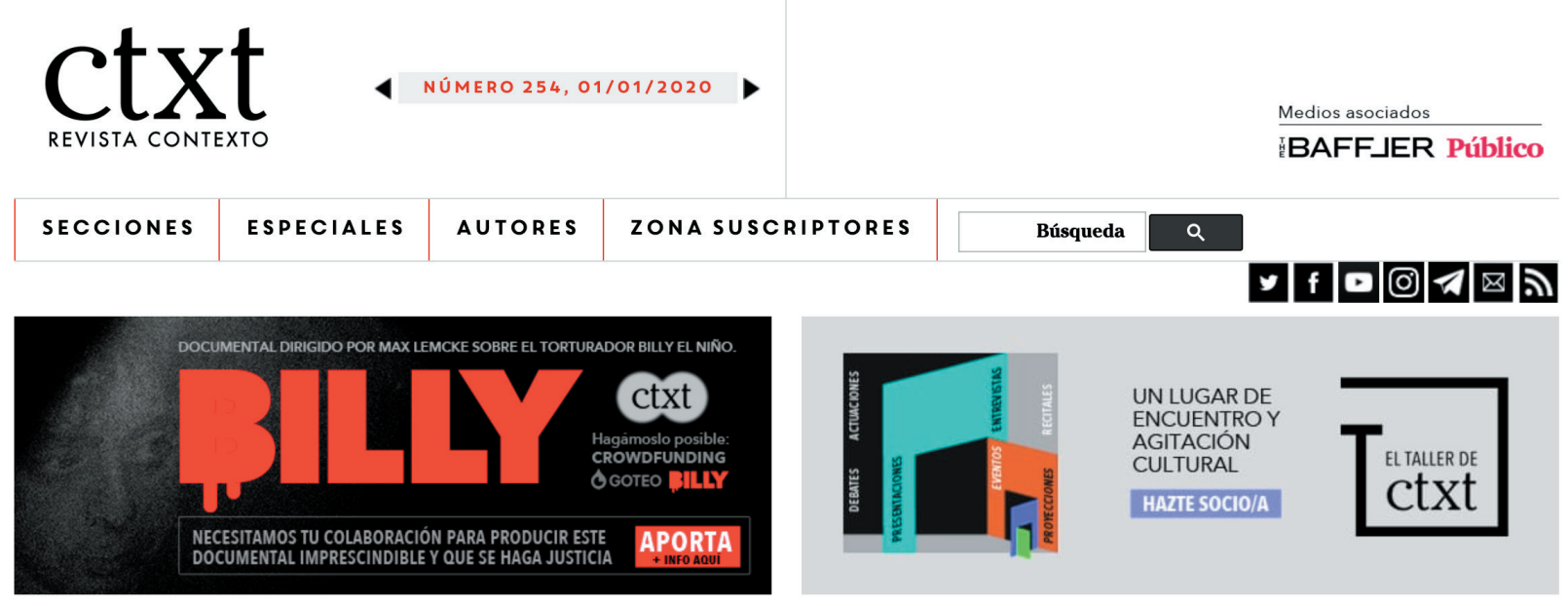

https://ctxt.es

El análisis que aquí presentamos deriva de un trabajo de campo en curso de corte cualitativo y etnográfico sobre CTXT. EI enfoque etnográfico está permitiendo aproximarnos al objeto de estudio desde el análisis de las prácticas y discursos de los mismos actores en su actividad cotidiana, con métodos como la observación directa, la participación del investigador en el contexto estudiado, la realización de entrevistas en profundidad (Hammersley; Atkinson, 1994; Guber, 2004) y el seguimiento de la actividad online (Hine, 2004; Lovink, 2011).

Nuestro interés por realizar una investigación etnográfica en CTXT se orienta a entender cómo los profesionales involucrados dan sentido a lo que hacen en el cambiante campo del periodismo digital (Deuze, 2017). Esto requirió de varias conversaciones y explicaciones que contaron siempre con una actitud abierta y colaboradora por parte de las tres personas fundadoras y responsables del medio, en las que centramos nuestra observación. Se discutieron y negociaron los roles, objetivos y límites que como investigadores nos correspondían (Guber, 2004; Kawulich, 2005). Como participante-observador (y siempre aclarando sus fines de investigación) una persona de nuestro equipo ha asistido a las sesiones del Consejo de redacción de CTXT al menos una vez al mes durante 3 años y a su grupo de WhatsApp. Otro de nosotros ha sido admitido como observador-participante en la sala de redacción de CTXT (septiembre de 2018) y en algunos de sus grupos internos de WhatsApp (enero de 2019), lo cual nos ha permitido conocer de primera mano

CTXT ha contado con el compromiso y la contribución económica de sus socios fundadores, así como con el apoyo económico y el interés de sus lectores y suscriptores la gestión del día a día del trabajo periodístico del medio, tanto en el espacio real o físico como en el espacio online, los cuales consideramos como formando un mismo espacio o contexto de investigación (Ardèvol, 2013).

En cuanto a las entrevistas, realizamos una primera tanda de entrevistas extensas por separado con las citadas tres personas fundadoras y actuales cargos directivos de CTXT en abril de 2018 y una segunda serie en enero de 2019, ésta enteramente focalizada a la cuestión del modelo de negocio. Las entrevistas han resultado centrales para este trabajo, pues constituyen una eficaz forma de identificar la memoria de unos sujetos, su experiencia y visiones en un momento determinado (García-Espín, 2016).

¿Qué puede aportar la etnografía al conocimiento del modelo de negocio de un medio digital nuevo? Como veremos, al hablar de modelo de negocio nos referimos a los instrumentos que el medio utiliza para organizar los recursos, competencias y actividades con el fin de crear valor. La investigación etnográfica nos permite indagar los fines y los valores de los actores y la forma en que los actualizan en su práctica, y acercarnos así a aspectos fundamentales de estos procesos de creación de un nuevo medio periodístico.

\section{Transformación de los modelos de negocio}

La discusión sobre los modelos de negocio en el ecosistema mediático actual se ha convertido en un debate recurrente en el contexto académico y profesional (Rojo-Villada, 2008; Casero-Ripollés, 2010; García-Avilés; González-Esteban, 2012; Vara-Miguel; Díaz-Espina, 2012; Cook; Sirkkunen, 2013; García-Santamaría; Clemente-Fernández; López-Aboal, 2013; García-Santamaría; Pérez-Serrano; Maestro-Espínola, 2016; Wagemans; Witschge; Deuze, 2016; Deuze, 2017; Bittner, 2019). Como ha recordado Campos-Freire (2010; 2011), el concepto de modelo de negocio es tan antiguo como los propios orígenes del estudio científico de la gestión empresarial, pero ha resurgido con fuerza en los últimos años 
ante el cambio que ha supuesto la transformación de la sociedad de la información, la generalización de internet y, más recientemente, el auge de las redes sociales y las aplicaciones móviles.

El modelo de negocio puede definirse como la manera en que la empresa estructura su actividad y genera ingresos (Rojo-Villada, 2008). Como señala Campos-Freire, el concepto de modelo de negocio es

"mucho más que la síntesis comercial y productiva de una empresa o sector, es un instrumento de gestión estratégica que describe y sintetiza la organización de los recursos, competencias y actividades para crear valor" (Campos-Freire, 2010, p. 17).

El modelo de negocio es, además, parte del mensaje de la empresa que lo pone en marcha, puesto que los modelos

"afectan en gran medida a lo que se produce, cómo se produce/distribuye y cuál es el usuario final que lo consume" (Cerezo, 2017, p. 4).

En el actual contexto mediático, la crisis y sus consecuencias sobre la generación de ingresos ha obligado a una revisión y redefinición de los modelos de negocio que afecta tanto a los medios de comunicación tradicionales como a los nuevos medios digitales propios de la cibercultura. En los últimos años, la digitalización ha transformado los hábitos de comunicación y con ellos la cadena de valor en su conjunto, tanto en lo que se refiere al modelo de producción como al de distribución y, por lo tanto, al modelo de negocio, ante lo cual los medios tradicionales no han tenido otra opción más que reinventarse. En este último campo, tras años de pruebas y errores, no se ha encontrado un modelo de negocio universal y estable, sino una suerte de combinación de diferentes fuentes de ingresos (Cerezo, 2017).

El entorno de los cibermedios periodísticos que operan exclusivamente en internet (es decir, sin referente impreso, radiofónico o audiovisual) es generalmente muy precario y competitivo y muchas de las empresas que se ponen en marcha fracasan (Wagemans; Witschge; Deuze, 2016, p. 161). Como en el caso de los medios tradicionales que han tenido que reinventarse para adaptarse al ecosistema digital, no existe un único modelo de negocio rentable y estable para los cibermedios sino que resulta ser siempre una combinación de diferentes fórmulas y fuentes de ingresos entre las que se incluyen la publicidad, el patrocinio, la intermediación, la explotación de la información, la venta de productos, la suscripción, la franquicia, el crowdfunding, los videojuegos, la realidad aumentada, la venta de apps, etc. (Rojo-Villada, 2008; García-Avilés; González-Esteban, 2012; Canavilhas, 2015; Deuze, 2017).

\section{Hacia la construcción de un modelo de negocio}

\subsection{Los comienzos: trabajo gratuito, financiación participativa y campañas de crowdfunding}

"Pusimos primero el periódico y después la empresa". Esta frase, pronunciada por uno de los fundadores de CTXT durante una de las entrevistas de nuestro trabajo de campo, resume los intereses del medio durante sus primeros años de vida y las dificultades a la hora de pensar en él en términos de empresa. De hecho, sus fundadores reconocen que no tenían "ni idea de empresa ni de qué negocio íbamos a hacer", por lo que el modelo de negocio se tuvo que ir improvisando sobre la marcha:

"Nosotros sabíamos de periodismo, pero no sabíamos gestionar una empresa, no sabíamos de marketing, no sabíamos de gerencia, no sabíamos de cuentas, en fin, es un proyecto hasta cierto punto un poco loco, de periodistas, y poco a poco le hemos ido dando una cierta estructura empresarial" (Fundador/a A).

Sí creían, sin embargo, que podían aportar un valor como medio:

"Nuestro modelo es la prensa anglosajona y la americana también. La idea de CTXT es recuperar las mejores cosas de la mejor tradición periodística, es decir: las buenas entrevistas, los buenos reportajes, los buenos editoriales, géneros que en internet habían desaparecido, estaban desapareciendo (...) Y ese rescate de géneros es también un planteamiento ético, es decir, esos géneros ayudan a clarificar en un momento de mucha confusión [...] Tenemos que ser complementarios a todos esos (medios digitales), el complemento de calidad y de reflexión que a ellos les falta (Fundador/a B).

CTXT se fundó como tal el 15 de enero de 2015 gracias a 14 periodistas que aportaron un capital inicial de 20.000 euros y que se comprometieron a trabajar gratis durante el primer año en las tareas de edición y redacción del medio hasta que la situación de su pequeña empresa les permitiera tener un salario. Comenzaron a trabajar en un medio que ni siquiera contaba con una redacción física, sino que el trabajo se realizaba en casa de cada uno y en el salón de casa de uno de sus socios fundadores:

"La empresa era tan minúscula que es que no había empresa: no había nóminas, no había inversión en tecnología, no había mobiliario, no había redacción, es decir, no había nada. Había una casa y catorce periodistas trabajando" (Fundador/a B).

Las redes interpersonales del grupo fundador y su experiencia previa en diarios como El país o El mundo les permitieron contactar con las personas que necesitaban para impulsar el nuevo medio y empezar a llenarlo de contenido y funcionalidad 
Junto al capital simbólico aportado por esos 14 competentes profesionales contaban con un importante capital social: las redes interpersonales del grupo fundador y su experiencia previa en diarios como El país o El mundo, que les permitieron contactar con las personas que necesitaban para impulsar el nuevo medio y empezar a llenarlo de contenido y funcionalidad. Gracias a ello se logró tejer una red de más de cien colaboradores altamente cualificados dispuestos a apostar por este medio en ciernes, entre los que se encontraban periodistas, analistas, editores, viñetistas, ilustradores, fotógrafos e informáticos. Nos hemos referido al trabajo "distribuido", no localizado en una redacción física, para la que no había en estos comienzos dinero, sino distribuido en diferentes lugares conectados al equipo central. Se trataba de una forma, entonces todavía precaria, del actual periodismo de equipos siempre conectados, o periodismo networked (Deuze, 2017). El diseño de la revista fue un asunto central y se resolvió en forma original y atractiva tomando como inspiración el diseño visual de la revista estadounidense The New Yorker.

El primer problema para el grupo fundador fue cómo hacer frente a los pagos por el trabajo de los colaboradores. El crowdfunding, por aquel entonces un mecanismo colaborativo de moda para la financiación de proyectos, se presentó como una posible solución:

"decidimos hacer un crowdfunding antes de haber salido, lo cual era muy arriesgado porque tú estás diciendo 'pon dinero aquí, que es una web que va a salir...'. Hicimos un vídeo muy bonito, contamos nuestra filosofía, qué es lo que iba a ser aquello, y la verdad es que funcionó, conseguimos el dinero que pedimos” (Fundador/a A).

La campaña se inició antes de la salida del primer número y en la descripción del proyecto ya quedaron sentados los principios editoriales del nuevo medio, en los cuales puede verse la importancia de la ideología ocupacional del periodismo tradicional como recurso en la nueva ola de periodismo emprendedor, tal y como han demostrado Wagemans, Witschge y Deuze (2016):

"Los fundadores de CTXT sabemos que sin prensa libre no hay democracia. Y pensamos que el actual descrédito de la prensa de referencia se debe, sobre todo, a su alianza con el poder político y económico. En CTXT queremos recuperar el viejo espíritu de la buena prensa independiente: ser un servicio público, escribir para la ciudadanía desconfiando de las verdades oficiales y de la propaganda que pone en circulación el poder. Nuestro lema será contexto y acción porque queremos ayudar a que la gente se forme un criterio propio para que pueda actuar de forma responsable y coherente".

https://www.verkami.com/projects/10753-ctxt

"El viejo espíritu de la buena prensa independiente", "servicio público", "para la ciudadanía", desconfiar de "las verdades oficiales y del poder". Estos son los argumentos principales con los que CTXT quiere atraer a una audiencia que espera sea sensible a ellos. Son también las razones, o los valores, por los que un buen grupo de periodistas y otros profesionales colabora con este medio, sin cobrar o con una retribución escasa y de futuro incierto. Esto presupone un malestar de al menos una parte de los periodistas y del potencial público con los medios periodísticos convencionales, que se detecta en España y en muchos otros lugares del mundo.

"Uno de los principales argumentos de compra (selling point) del periodismo profesado por Mediapart es el que desafía y aporta una alternativa a la prensa mainstream francesa".

Una alternativa enraizada en el ideal del viejo buen periodismo (Wagemans; Witschge; Deuze, 2016, p. 175). En la hipótesis de CTXT, ese malestar latente en el público se une a una disposición activa a contrarrestarlo, a participar en proyectos que proponen una alternativa. Una importante red de profesionales se pone en marcha para captar a esa audiencia prevista.

La campaña de crowdfunding se resolvió con éxito y, gracias a ello, el medio pudo cumplir el primer objetivo que se había marcado: pagar durante los primeros tres meses el trabajo de los colaboradores de la publicación (sobre todo a los informáticos, imprescindibles para asegurar la funcionalidad del medio en red, que "se llevaron casi todo el dinero que conseguimos"). El crowdfunding fue una salida para garantizar la sostenibilidad de la publicación durante sus primeros meses, coincidiendo con una época en la que este sistema de financiación alternativo se estaba convirtiendo en una interesante

"vía de arrancar un proyecto o de testar si el producto es interesante antes de lanzarlo e invertir recursos",

o lo que es lo mismo,

"una forma de poner en contacto la energía emprendedora del creador con la demanda interesada de los usuarios" (Martínez-Sánchez; Martínez-Polo, 2017, pp. 227-228).

Para los fundadores de CTXT, el crowdfunding, además de dar a conocer el proyecto e incorporar a más público, fue la alternativa para lograr financiación evitando a los bancos u otras entidades o instituciones que acabaran comprometiendo su libertad editorial:

“Nosotros no podíamos, por ejemplo, acudir a un fondo de capital [...] no estábamos en la disposición de asegurar ninguna rentabilidad porque era una cosa muy precaria y hecha con muy poco dinero. Desde luego, habíamos decidido no pedir ningún préstamo a los bancos para no depender de nadie" (Fundador/a A).

Ante los buenos resultados conseguidos durante la primera campaña, CTXT ha recurrido en otras ocasiones al crowd- 
funding como vía de financiación y parte de su modelo de negocio. En su segundo año, en febrero de 2016, sus fundadores iniciaron una nueva campaña en la plataforma Goteo.org que ellos denominan "crowdfunding de consolidación" y en la que se pedía financiación a los lectores para lograr consolidar el proyecto. La campaña, titulada El contexto lo es todo, volvía a destacar la filosofía editorial de la publicación, incidiendo en la necesidad de realizar un "periodismo de contexto" y de servicio público.

https://www.goteo.org/project/ctxt

Esta nueva campaña obtuvo una financiación de 72.883 euros de un presupuesto mínimo de 52.000 euros y óptimo de 77.000, dinero que se utilizó nuevamente para pagar las colaboraciones e incluso subir su importe, permitiendo así "crear un equipo sólido de trabajo que pueda vivir de su trabajo" y apoyar la independencia de la publicación.

Los fundadores de CTXT han ido descubriendo la posibilidad de la financiación participativa, que consideran un modelo de negocio "muy sano":

"Creo que es importante que los lectores hayan confiado en que el proyecto empresarial era viable. Y yo creo que ese es un poco el modelo que decía Julia Cagé que había que poner en marcha. Y además es muy sano que tengamos una empresa en la que todos los socios son lectores o colaboradores" (Fundador/a B).

CTXT ha publicado varios artículos de Julia Cagé, economista doctorada en Harvard, quien sostiene que

"La información es un bien público que debe ser accesible a todos. Y por ello hay que proteger su producción, lo que hoy significa repensarla" (Cagé, 2016, p. 27).

Esta autora defiende la "financiación participativa" de los medios independientes y propone

"favorecer la multiplicidad de accionistas y evitar un exceso de concentración en manos de una o dos personas" (Ralle-Andreoli, 2016).

En este sentido, la sociedad CTXT ha realizado varias ampliaciones de capital a lo largo de sus cuatro años de existencia:

"Hemos ido sumando socios, hemos abierto la empresita, tiene ahora 140 o 150 socios, entonces cada año hemos ido haciendo ampliaciones de capital populares para que la gente que nos leía y nos apoyaba y se suscribía pudiera ser también accionista. Y eso es lo más interesante del proyecto, yo creo, que hay 150 personas dispuestas a poner el dinero" (Fundador/a B).

Así, si como han señalado autores como Canavilhas (2015), los nuevos medios deberían implementar estrategias de lealtad basadas en la valoración de una red propia que pueda involucrar al usuario y crear sentimientos de pertenencia (ofreciéndoles, por ejemplo, la posibilidad de interactuar con sus periodistas, participar en foros internos, etc.), en el caso de CTXT este sentimiento de pertenencia se construye y se impulsa no sólo mediante la interacción con los periodistas y la participación en foros y encuentros, sino también ofreciendo a sus lectores la posibilidad de convertirse en accionistas del medio y ser parte activa de él.

\subsection{El modelo de suscripciones}

En octubre de 2016, unos meses después de la finalización de la campaña de crowdfunding de Goteo.org, el grupo fundador de CTXT decide experimentar con otra vía de financiación: las suscripciones. Sus fundadores entendían que el periodismo de "servicio público" implica la accesibilidad total y gratuita de los contenidos, por lo que tuvieron que pensar un modelo de suscriptores que pagaran por algo que es gratis, porque "nunca nos planteamos cerrar la página, o sea, hacer el modelo de InfoLibre, hacer un muro de pago". De ahí que, al modo de las campañas de crowdfunding llevadas a cabo anteriormente, el grupo fundador, además de apelar al valor del medio y a la libertad de prensa, propusiera distintos tipos de "recompensas" para que los lectores estuvieran dispuestos a pagar por un medio esencialmente gratuito: acceso a espacios y contenidos sólo para suscriptores, tazas, camisetas, bolsas de tela, regalo de entradas, etc.

Las suscripciones se han convertido en la principal fuente de ingresos de CTXT, gracias a la cual en diciembre de 2017 la revista logró alcanzar el hito de los 5.000 suscriptores, que aportaron el 53\% de los ingresos totales. A día de hoy, el número de suscriptores ha aumentado hasta los 7.500 y en 2018 supuso la fuente principal de ingresos, el 33\%, si bien el objetivo final del medio para cubrir los gastos de la empresa era de 10.000 suscriptores.

El modelo de suscripciones ha ido variando desde su implantación en octubre de 2016 y en la actualidad cuenta con tres tipos de suscripción anual que incluyen acceso a "El saloncito" (una zona de contenido exclusivo para suscriptor@s, con videos de entrevistas a pensadores estadounidenses, El tintero de CTXT, Eventos CTXT, Libros CTXT, etc.), carta semanal en exclusiva y adelantos editoriales. Durante su rutina semanal, los responsables del medio dedican varias horas a la gestión de las suscripciones y el contacto directo con los suscriptores, que son tratados como "defensores" del periodismo libre e independiente que postula CTXT. Requiere unos suscriptores peculiares, que han de ser efectivamente "defensores" de la "causa" CTXT, en la que participan con su aportación económica. El crowdfunding de consolidación y las suscripciones conseguidas (sumadas al resto de fuentes de financiación que mencionaremos) permitieron alquilar 
en 2017 un local para la redacción y estabilizar una plantilla mínima. A mediados de 2018, afirma uno de los fundadores,

"hemos subido apreciablemente las tarifas de los colaboradores y nos hemos puesto un sueldo digno para poder pagar el alquiler. Y hoy estamos contratando a tres jóvenes periodistas en prácticas" (Fundador/a B).

A finales de ese año 2018, CTXT pagaba regularmente siete sueldos a periodistas. Para ello ha tenido que encontrar otras fuentes de ingresos.

\subsection{La cesión de tráfico}

Junto al modelo de suscripciones, otra vía de financiación paralela con la que CTXT logra actualmente parte de sus ingresos es la cesión de tráfico. Sus fundadores reconocen que esto es algo que hacen los medios pequeños para lograr una mayor visibilidad. El diario digital Público contactó con CTXT para ofrecerles un contrato de cesión de tráfico, contrato que actualmente mantienen y que supone en torno al $11 \%$ de sus ingresos. Sin embargo, aunque reconocen la afinidad entre ambos medios en línea editorial, los responsables de CTXT son críticos con este contrato de cesión de tráfico, pues, afirman, "nos canibaliza un poco la marca":

"Si pudiéramos no tener esa cesión de tráfico, porque nos canibaliza un poco la marca, pues no lo haríamos. Nosotros cedemos nuestros lectores, es decir, nuestros números, ellos obtienen más publicidad y a nosotros nos llega una parte, que supone eso, el $11 \%$ de nuestros ingresos. También supone que los contenidos que nosotros les mandamos a ellos aparezcan en su portada y es verdad que como Público es un medio muy masivo, nuestros contenidos se leen más. Eso tiene la parte buena de que se nos ve más, pero tiene la parte mala de que hay gente que cree que CTXT forma parte de Público" (Fundador/a A).

"Público es un periódico de audiencia masiva y de última hora, hacen poco análisis, y nosotros somos un medio mucho más reposado, mucho más analítico" (Fundador/a A),

de ahí que el lema de la publicación sea "Orgullosas de llegar tarde a las últimas noticias". Durante 2018 se realiza un pequeño cambio en el diseño de la cabecera, de modo que el logo de Público no aparece ya aislado, debajo del logo de CTXT, sino unido al de The Buffler -una revista americana de crítica cultural y política con la que el medio comparte artículos-, bajo el epígrafe "Medios asociados", lo que ha reducido el peligro de confusión y de "canibalización de la marca".

\subsection{La publicidad}

Aunque los responsables de CTXT mantienen un discurso crítico con la publicidad "cuando condiciona la línea editorial", ésta supone otra parte de sus ingresos. En su rendición de cuentas del año 2017, la publicidad supuso en torno al $14 \%$ de los ingresos de la publicación, y en el 2018 el 11,9\%. Para ellos, la publicidad cumple un papel "residual" o "marginal" dentro de su modelo de negocio, y señalan que

"probablemente seamos el medio que menos banners tiene por centímetro cuadrado" (Fundador/a A).

La filosofía del medio -crítico con el mercado publicitario actual, basado en la lógica del "pinchazo" o clickbait- y su formato de semanario lo hacen poco atractivo para los anunciantes y agencias de publicidad:

"Nosotros no garantizamos ningún tipo de tráfico ni de audiencia, ni de clicks, ni nada de eso, porque es imposible" (Fundador/a C).

"Nosotros no vamos a bulto. Al ser un medio que no va a volumen y no hacemos contenido patrocinado, entonces no interesamos ni a los anunciantes ni a las agencias. Somos como la migajilla" (Fundador/a B).

Los ingresos derivados de la publicidad provienen principalmente de empresas e instituciones públicas como ayuntamientos o museos. También bancos como Bankia o Ibercaja han aportado algo, pero muy poco -"una miseria"- lo que no les impide, por ejemplo, informar sobre los juicios de Rato u otros asuntos relacionados con los bancos:

"La ventaja de tener tan poca publicidad es que eres libre para escribir sobre todas esas empresas" (Fundador/a B).

Por último, aunque el porcentaje y visibilidad de la publicidad en CTXT es pequeño comparado con el de muchos otros medios informativos españoles, sus responsables no son partidarios de eliminarla por completo pues consideran que

"un medio que no tenga publicidad, ninguna publicidad en portada, parece que es un medio que esté moribundo, que no está en el mercado" (Fundador/a B).

\subsection{Otras vías de financiación}

A las vías de financiación señaladas anteriormente se unen otras fuentes de ingresos de diversa procedencia entre las que destacaremos, en primer lugar, el patrocinio de empresas e instituciones. En este sentido, los entrevistados señalan que el patrocinio de La Caixa es para dar noticias sociales que a la Fundación La Caixa le interesa difundir, pero que son elegidas por los propios responsables del medio:

La filosofía del medio -crítico con el mercado publicitario actual, basado en la lógica del "pinchazo" o clickbait- y su formato de semanario lo hacen poco atractivo para los anunciantes y agencias de publicidad 
"No es contenido patrocinado, sino que es contenido que nosotros elegimos y decidimos Es una especie de publicidad indirecta, pero no hay ninguna sumisión editorial".

Además, señalan, este tipo de contenido es coherente con la línea editorial que CTXT ha seguido desde el principio:

"son temas sociales, temas de exclusión, temas de educación, en fin, temas que tocan asuntos que nosotros ya estábamos haciendo" (Fundador/a B).

Otra fuente adicional de ingresos es el denominado El dobladillo, una publicación en papel en formato desplegable inspirada en la publicación francesa Le 1 y a la que el medio se refiere significativamente como "el papel interactivo y ecológico de CTXT". Esta publicación impresa nació como un regalo para los suscriptores, aunque actualmente se vende también de forma separada y suele llevar piezas rescatadas de la web, así como piezas totalmente nuevas.

Los eventos Fuera de contexto son otra fuente de ingresos. Se trata de una serie de entrevistas a personajes del mundo de la política y las artes que la publicación realiza cada cierto tiempo en el Teatro del Barrio de Lavapiés. Para los responsables de CTXT, estos eventos son, junto con El dobladillo,

"una forma de tener una presencia física en la calle" y señalan que "nos dan algo de dinero, es una pequeñita vía de financiación, pero no lo hacemos tanto por el dinero que nos da sino por el contacto directo con la gente", los lectores actuales y potenciales (Fundador/a A).

CTXT ha fundado además la Colección contextos, con la editorial Lengua de Trapo, con la que a comienzos de 2019 lleva publicados 5 libros, lo cual ha supuesto otra vía de ingresos,

"en la que ellos (Lengua de Trapo) invierten el capital (y aportan el trabajo editorial) y nosotros ponemos los autores de CTXT, la marca y la difusión en redes y la venta online" (Fundador/a B).

Este medio ha establecido también acuerdos de intercambio de artículos con otros medios periodísticos digitales: Social Europe, The baffler, Political critic, Inpiù, Italy, El estornudo..., con los que CTXT participa en la formación de redes de personas, espacios y recursos típica de estas nuevas formas de periodismo (Deuze, 2017). De esta forma, cuenta con piezas de estos medios a cambio, no de dinero, sino de poner a su disposición artículos de CTXT.

CTXT experimenta continuamente con otras fórmulas con las que lograr nuevos ingresos que permitan sostener su independencia. En este sentido, el medio ha organizado las I Jornadas internacionales feministas, celebradas los días 8 y 9 de noviembre de 2018 en la ciudad de Zaragoza, que han permitido a CTXT obtener una subvención de la fundación Open Society para su celebración, y que, con sus packs de suscripción, venta de gadgets, etc., supuso en torno al 7\% de los ingresos anuales de ese año.

Todo ello ha llevado a CTXT a tener un elevado número de lectores y una influencia, afirman, en la agenda pública:

"La influencia se mide en la cantidad de temas que nosotros hemos metido en la agenda que no estaban (...) Hemos tenido posturas claras en muchos temas que la prensa mainstream no estaba tratando o estaba ignorando" (Fundador/a B).

"Hay 600.000 personas todos los meses que se meten, son 120.000 a la semana, o sea 120.000 por número que leen 12 minutos de media cada vez que entran y eso es mucho. Para un país como este donde no hay tampoco tradición de leer mucho, yo creo que sí, que hay un nicho de lectores que necesitaba esto claramente" (Fundador/a B).

\section{Conclusiones}

La construcción de un modelo de negocio sostenible y estable es uno de los grandes retos a los que se enfrentan actualmente los medios digitales. Después de varios años de existencia de la cultura digital, los cibermedios siguen desarrollando un alto grado de experimentación e investigación de fórmulas con las que lograr la financiación necesaria para hacer viables sus proyectos informativos (Rojo-Villada, 2008; García-Avilés; González-Esteban, 2012; García-Santamaría; Pérez-Serrano; Maestro-Espínola, 2016; Deuze, 2017; Bittner, 2019).

En el caso de CTXT hemos visto cómo la combinación de diferentes fuentes de ingresos, desde las campañas de crowdfunding hasta los ingresos por suscripciones, cesión de tráfico, publicidad o patrocinio, ha generado un modelo de negocio híbrido que está en transformación permanente y que no termina de asentarse totalmente, algo que sus responsables relacionan no sólo con su propia experiencia sino con la situación global del periodismo digital:

“El modelo de negocio de los digitales está en construcción, está en transformación permanente y va pareciendo cada vez más claro que la independencia real sólo se va a conseguir si hay una masa suficiente de suscriptores que te permitan no depender de la publicidad o de otras formas de presión política como es la publicidad institucional" (Fundador/a B).

Es siempre difícil conseguir la masa de suscriptores necesaria para cubrir todos los gastos de una empresa periodísti$\mathrm{ca}^{1}$. El modelo de negocio de CTXT está condicionado por el principio (que podemos llamar ético o ideológico) de no cobrar por contenidos, no hacer "muro de pago" sólo para suscriptores, como hacen muchos otros digitales, lo que incrementa esa dificultad, pues los suscriptores de CTXT han de pagar por algo que es básicamente gratuito. El equipo 
está atento a la fluctuación del número de lectores y de suscriptores-defensores, que señalan el acierto o el fracaso del medio para convencerlos en cada número y son el motivo para seguir esforzándose en lograr su objetivo. Siguiendo una estrategia común a la mayoría de las actuales start-ups periodísticas digitales en todo el mundo, reducen todo lo posible los costes de producción y combinan diferentes fuentes de ingresos. Entre éstas se encuentran algunas que suponen decisiones difíciles y en cierto modo contradictorias con la filosofía editorial de CTXT, como la inclusión de publicidad de bancos (aunque mantenida en un porcentaje mínimo que no condicione su línea editorial) o la cesión de tráfico a un medio afín en cuanto a su línea editorial, pero muy distinto a ellos, como Público.

En cuanto a la reducción de costes, además de por el aprovechamiento, a menudo creativo, de las condiciones que permite la tecnología digital (el acceso a la información y al público, la producción en red de equipos conectados a distancia, etc.), CTXT ha sido posible en buena parte gracias a la pasión por el buen periodismo y al trabajo "atípico" (Deuze, 2017) de periodistas muy cualificados, que ha sido gratuito por parte de 14 de ellos durante el primer año y de tres durante dos años:

"El concepto de trabajo no encaja con esto, porque entonces pareceríamos locos o estaríamos enfermos. Es que esto es un modo de vida, es nuestra prioridad vital" (Fundador/a C).

El entusiasmo que potencia este capital simbólico, unido al capital social formado por las redes interpersonales y digitales tejidas por este medio y por sus profesionales, ha sido fundamental para realizar un periodismo exigente, crítico y de calidad que ha sabido encontrar -y también crear- su nicho de audiencia.

Los fundadores de CTXT están convencidos de que su publicación es un modelo periodístico necesario y posible, un modelo "casero" (do-it-yourself) pero "sano" y sostenible, en el sentido de que con muy poco dinero se ha llegado muy lejos:

"Yo creo que CTXT es un modelo posible para crear medios digitales. Creo que no ha habido un medio que haya salido al mercado con menos dinero que este. CTXT sirve para que se entienda que un medio de internet no necesita una gran inversión. Con una mínima inversión y el compromiso de un grupo de periodistas suficientemente amplio se puede crear un medio y además se puede crear un medio de calidad y un medio que no tenga casi competencia, porque si eliges bien tu nicho eso te va a permitir distinguirte de los otros" (Fundador/a B).

A comienzos de 2019, aunque la estabilidad parece lograda y el semanario se enorgullece de haber cerrado el 2018 con unos 30.000 euros de beneficios, "sin un euro de deudas, ni un favor debido a nadie poderoso", la búsqueda de recursos económicos sigue siendo un problema con el que el equipo de CTXT tiene que lidiar cotidianamente.

\section{Notas}

1. El francés Mediapart, digital que se promueve como desafío y alternativa a la prensa convencional, fue pionero en el modelo de suscripciones, gracias al cual se mantiene (Wagemans; Witschge; Deuze, 2016). Sin embargo, como reconoce Bittner (2019), aunque algunos periódicos pueden conseguir ingresos significativos mediante el modelo de suscripciones, incluso medios de comunicación importantes como The New York Times no pueden ser sostenidos únicamente mediante suscripciones, lo cual hace necesario la búsqueda continua de nuevas fuentes de ingresos.

\section{Referencias}

Ardèvol, Elisenda (2013). Cultura digital y prácticas creativas. Tientos etnográficos en torno a la cultura libre. Barcelona: IN3-Working Paper Series.

https://doi.org/10.7238/IN3WPS.VOI0.1817

Bittner, Andreas K. (2019). Digital journalism \& new business models. European Federation of Journalists. https://europeanjournalists.org/blog/2019/02/25/efj-study-on-new-business-models-to-promote-journalism

Cagé, Julia (2016). Salvar los medios de comunicación. Barcelona: Anagrama. ISBN: 9788433963963

Campos-Freire, Francisco (2010). "Los nuevos modelos de gestión de las empresas mediáticas". Estudios sobre el mensaje periodístico, v. 16, pp. 13-30.

https://revistas.ucm.es/index.php/ESMP/article/view/ESMP1010110013A

Campos-Freire, Francisco (2011). "Las empresas de medios de comunicación revisan y amplían sus modelos de negocio". Razón y palabra, n. 74.

http://www.razonypalabra.org.mx/N/N74/VARIA74/16FreireV74.pdf

Canavilhas, João (2015). “Nuevos medios, nuevo ecosistema”. El profesional de la información, v. 24, n. 4, pp. 357-362. https://doi.org/10.3145/epi.2015.jul.01 
Casero-Ripollés, Andreu (2010). “Prensa en internet: nuevos modelos de negocio en el escenario de la convergencia”. EI profesional de la información, v. 19, n. 6, pp. 595-601.

https://doi.org/10.3145/epi.2010.nov.05

Cerezo, Pepe (2017). En busca del modelo de negocio. Madrid: Evoca Comunicación e Imagen.

http://evocaimagen.com/dosieres/dosier-evoca-06-modelos-de-negocio.pdf

Cook, Clare E.; Sirkkunen, Esa (2013). "What's in a niche? Exploring the business model of online journalism". Journal of media business studies, v. 10, n. 4, pp. 63-82.

https://doi.org/10.1080/16522354.2013.11073576

Deuze, Mark (2017). “Considering a possible future for digital journalism”. Revista mediterránea de comunicación, v. 8, n. 1, pp. 9-18.

https://doi.org/10.14198/MEDCOM2017.8.1.1

Deuze, Mark; Witschge, Tamara (2017). "Beyond journalism: Theorizing the transformation of journalism”. Journalism, v. 19, n. 2, pp. $165-181$.

https://doi.org/10.1177/1464884916688550

Fernández-Sande, Manuel (2013). "La crisis de la prensa en España: análisis del caso Público, un diario nacido a contracorriente". Vivat academia, n. 122, pp. 22-39.

https://doi.org/10.15178/va.2013.122.22-39

García-Avilés, José-Alberto; González-Esteban, José-Luis (2012). “Cibermedios nativos españoles: explorando modelos de rentabilidad". Trípodos, n. 30, pp. 153-167.

http://www.tripodos.com/index.php/Facultat_Comunicacio_Blanquerna/article/view/50

García-Espín, Patricia (2017). “Etnografía y ciencia política: la excepcionalidad del caso español”. Política y sociedad, v. 54, n. 1, pp. 249-269.

https://doi.org/10.5209/POSO.48938

García-Orosa, Berta (2016). “Eldiario.es: un modelo de negocio mixto”. En: Sábada, Charo; García-Avilés José-Alberto; Martínez-Costa, María-del-Pilar (coords.). Innovación y desarrollo de los cibermedios en España. Pamplona. Eunsa. ISBN: 9788431331252

García-Santamaría, José-Vicente; Clemente-Fernández, María-Dolores; López-Aboal, María (2013). “La organización de las redacciones en los nuevos diarios digitales españoles y su relación con los nuevos modelos de negocio". Textual \& visual media, n. 6, pp. 141-160.

http://textualvisualmedia.com/index.php/txtvmedia/article/view/81

García-Santamaría, José-Vicente; Pérez-Serrano, María-José; Maestro-Espínola, Lidia (2016). “Los clubs de suscriptores como nuevo modelo de financiación de la prensa española”. El profesional de la información, v. 25, n.3, pp. 395-403. https://doi.org/10.3145/epi.2016.may.09

González-Esteban, José-Luis (2014). “La transformación del ecosistema mediático español: el caso de eldiario.es”. Revista mediterránea de comunicación, v. 5, n. 2.

https://doi.org/10.14198/MEDCOM2014.5.2.10

Guber, Rosana (2004). La etnografia. Método, campo y reflexividad. Buenos Aires: Norma. ISBN: 9789876291576

Hammersley, Martyn; Atkinson, Paul (1994). Etnografía. Métodos de investigación. Barcelona: Paidós. ISBN: 97884 49309809

Hine, Christine (2004). Etnografía virtual. Barcelona: Editorial UOC. ISBN: 8497880196

Kawulich, Barbara B. (2005). "La observación participante como método de recolección de datos". Forum: Qualitative social research, v. 6, n. 2, art. 43.

http://diverrisa.es/uploads/documentos/LA-OBSERVACION-PARTICIPANTE.pdf

López-García, Xosé (2011). La metamorfosis del periodismo. Sevilla: Comunicación Social. ISBN: 9788492860692

López-García, Xosé (2016). “InfoLibre: periodismo independiente para crear una comunidad”. En: Sábada, Charo; García-Avilés, José-Alberto; Martínez-Costa, María-del-Pilar (coords.). Innovación y desarrollo de los cibermedios en España. Pamplona: Eunsa. ISBN: 9788431331252

Lovink, Geert (2011). My first recession. Critical internet culture in transition. Amsterdam. Institute of Network Cultures. ISBN: 9789056623531

Martínez-Sánchez, Jesús; Martínez-Polo, Josep M. (2017). "El Salto y su propuesta de periodismo cooperativo". Redes. com. Revista de estudios para el desarrollo social de la comunicación, n. 15, pp. 224-249.

http://193.147.26.104/handle/10952/3364 
Peñamarín, Cristina (2016). "Controversia e sfera pubblica. Il caso della crisi del giornalismo in Spagna". Sociologia della comunicazione, 51.

https://doi.org/10.3280/SC2016-051002

Ralle-Andreoli, François (2016). “Julia Cagé / Economista. Autora de 'Salvar los medios de comunicación'. 'Los medios pequeños deben convertir en accionistas a sus donantes'”. CTXT, 9 marzo.

https://ctxt.es/es/20160302/Politica/4689/Cag\%C3\%A9.htm

Rojo-Villada, Pedro-Antonio (2008). Modelos de negocio y consumo de prensa en el contexto digital. Murcia: Universidad de Murcia. ISBN: 9788483717448

Rubio-Jordán, Ana-Virginia (2014). "La aparición de InfoLibre y eldiario.es para la defensa de un periodismo más democrático y participativo". Historia y comunicación social, v. 19, n. esp. enero, pp. 491-500.

https://core.ac.uk/download/pdf/38816773.pdf

Vara-Miguel, Alfonso; Díaz-Espina, Carolina (2012). "Modelos de negocio y estrategia editorial: el caso del wsj.com”. Revista de comunicación, n. 11, pp. 110-126.

https://dialnet.unirioja.es/servlet/articulo?codigo $=4508615$

Wagemans, Andrea; Witschge, Tamara; Deuze, Mark (2016). "Ideology as resource in enterpreneural journalism. The French online news startup Mediapart". Journalism practice, v. 10, n. 2, pp. 160-117.

https://doi.org/10.1080/17512786.2015.1124732

\section{Colección de libros de bolsillo El profesional de la información (Editorial UOC) Últimos títulos publicados}
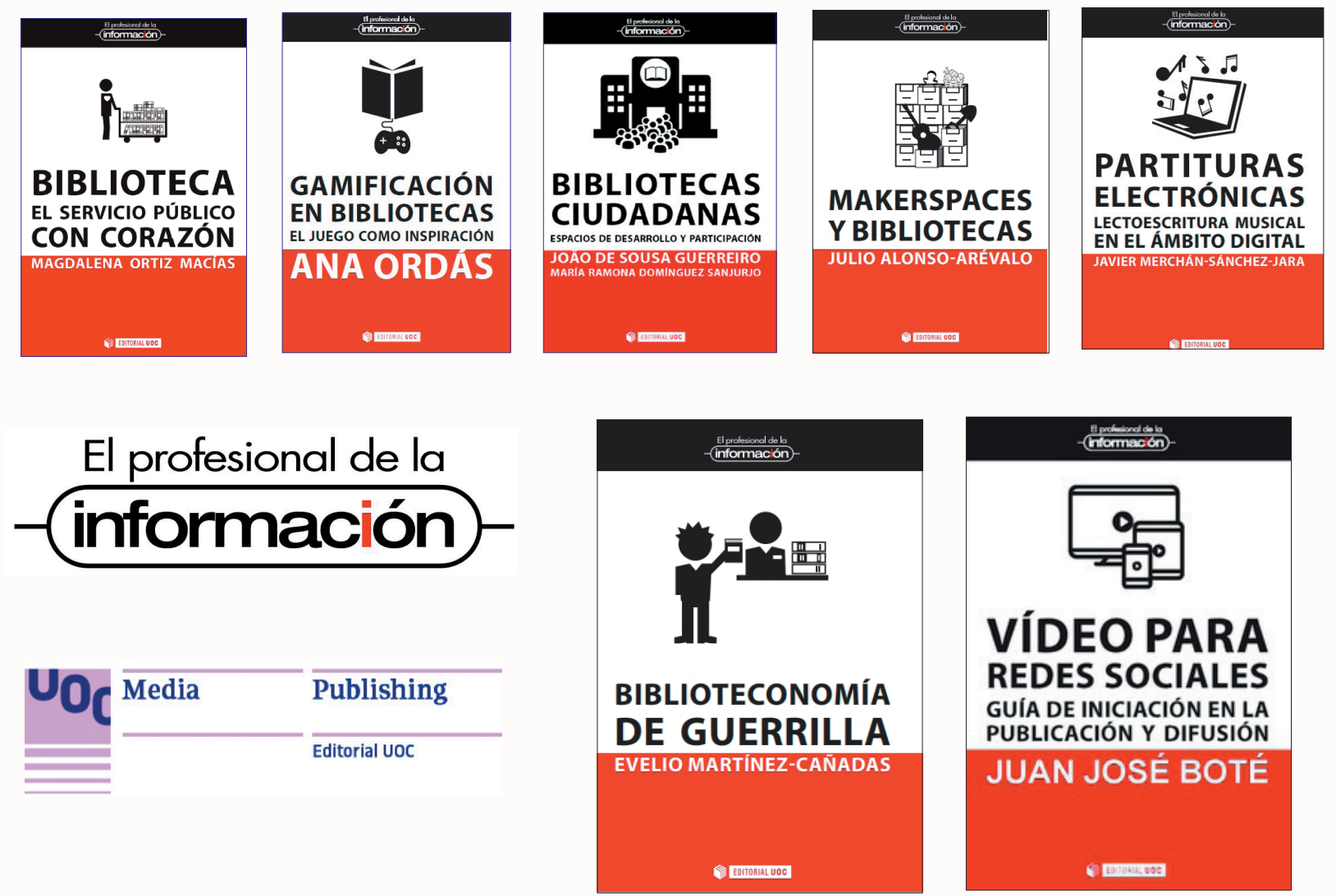

Más información:

http://www.elprofesionaldelainformacion.com/libros.html 